\title{
Role of the trigonal warping on the minimal conductivity of bilayer graphene
}

\author{
József Cserti, ${ }^{1}$ András Csordás, ${ }^{2}$ and Gyula Dávid ${ }^{3}$ \\ ${ }^{1}$ Department of Physics of Complex Systems, Eötvös University \\ H-1117 Budapest, Pázmány Péter sétány 1/A, Hungary \\ 2 HAS-ELTE, Statistical and Biological Physics Research Group, \\ H-1117 Budapest, Pázmány Péter sétány 1/A, Hungary \\ ${ }^{3}$ Department of Atomic Physics, Eötvös University \\ H-1117 Budapest, Pázmány Péter sétány 1/A, Hungary
}

(Dated: September 29, 2018)

\begin{abstract}
Using a reformulated Kubo formula we calculate the zero-energy minimal conductivity of bilayer graphene taking into account the small but finite trigonal warping. We find that the conductivity is independent of the strength of the trigonal warping and it is three times as large as that without trigonal warping, and six times larger than that in single layer graphene. Although the trigonal warping of the dispersion relation around the valleys in the Brillouin zone is effective only for low energy excitations, our result shows that its role cannot be neglected in the zero-energy minimal conductivity.

PACS numbers: 81.05.Uw, 73.23.Ad, 72.10.Bg, 73.43.Cd
\end{abstract}

Recent experiments have proved that the charge carriers in graphene (single or stacks of atomic layer of graphite) are massless Dirac fermions $1,2,3$. For recent reviews on graphene see Refs. 4, $, 5,6$. Besides the unusual transport properties observed and reviewed in the above works another important experimental feature is the minimal conductivity of the graphene systems which was considered theoretically ${ }^{\underline{7}}$ long before the experimental evidence. After the above mentioned experimental works on graphene, number of theoretical studies $8,9,10,11,12,13,14,15,16,17,18$ have predicted the conductivity of the order of $e^{2} / h$. Very recently, Miao et al. have experimentally confirmed ${ }^{19}$ most theoretical predictions $11,13,14,15,16,17,18$, namely the minimum conductivity in wide and short strips approaches the universal value $\sigma_{x x}^{\min }=(4 / \pi) e^{2} / h$ in single layer graphene.

The bilayer graphene has been studied first experimentally $\underline{\underline{3}}$ by Novoselov et al. and theoretically ${ }^{20}$ by McCann and Fal'ko. McCann have calculated the asymmetry gap in the electronic band structure of bilayer graphene ${ }^{21}$. In biased bilayer graphene it was demonstrated that the gap can be tuned by electric field effect 22 . In bilayer graphene the semiconductor gap has recently been controlled experimentally by Ohta et al $\stackrel{23}{ }$. The optical and magneto-optical far infrared properties of bilayer graphene has been studied by Abergel et al. 24 . The role of the impurities in biased bilayer graphene has been studied by Nilsson and Neto 25 . Ludwig has considered the conductance of a normal-superconductor junction in bilayer graphene ${ }^{26}$. Recently, Koshino and Ando have investigated the transport in bilayer graphene in self-consistent Born approximation 27 and they found that in the strong-disorder regime $\sigma_{x x}^{\min }=(8 / \pi) e^{2} / h$, while in the weak-disorder regime $\sigma_{x x}^{\min }=(24 / \pi) e^{2} / h$ which is six times larger than in single layer graphene. Similarly, Katsnelson has also calculated the minimal conductivity in bilayers using the Landauer approach 28 and he obtained a different value $\sigma_{x x}^{\min }=2 e^{2} / h$. In
Ref. 17 we found $\sigma_{x x}^{\min }=(8 / \pi) e^{2} / h$ which was confirmed later by Snyman and Beenakker 29 using the Landauer approach.

However, much fewer theoretical works paid attention to the role of the trigonal warping in bilayer graphene. The influence of the trigonal warping on the weak localization effect has been investigated by Kechedzhi et al $\stackrel{30}{ }$, while on the minimal conductivity only by Koshino and Ando $\frac{27}{2}$ using an effective 2 by 2 Hamiltonian. Our aim in this work is to calculate the minimal conductivity using the Hamiltonian suggested originally by McCann and $\mathrm{Fal}^{\prime} \mathrm{ko} \underline{\underline{20}}$. This Hamiltonian allows us to find the zero-energy minimal conductivity as a function of the strength of the trigonal warping in bilayer graphene. We use the Kubo formula rewritten in a form suitable for obtaining the zero-energy minimal conductivity in graphene systems. Surprisingly, we find that the conductivity is independent of the strength of the trigonal warping and six times as large as that for single layer graphene.

The bilayer graphene consists of two coupled honeycomb lattices with basis atoms $A_{1}, B_{1}$ and $A_{2}, B_{2}$ in the bottom and the top layers, respectively. The two layers are arranged in Bernal stacking $\left(A_{2}-B_{1}\right)$. The intralayer coupling between $A_{1}$ and $B_{1}$, and $A_{2}$ and $B_{2}$ is $\gamma_{0}$. The strongest interlayer coupling is between $A_{2}$ and $B_{1}$ with coupling constant $\gamma_{1}$. A direct hopping between $A_{1}$ and $B_{2}$ is taken into account by the coupling constant $\gamma_{3} \ll \gamma_{1}$. This coupling is responsible for the trigonal warping. The above coupling constants are estimated as $\gamma_{0}=3.16 \mathrm{eV}^{31}, \gamma_{1}=0.39 \mathrm{eV}^{32}$, and $\gamma_{3}=0.315 \mathrm{eV}^{33}$.

To model the bilayer graphene we use the same gapless Hamiltonian as that in Ref. 20 which takes into account the trigonal warping. The Hamiltonian in the basis $A_{1}, B_{1}, A_{2}, B_{2}$ in the valley $\mathbf{K}$ and in the basis 
$B_{1}, A_{1}, B_{2}, A_{2}$ in the valley $\mathbf{K}^{\prime}$ reads

$$
H_{b 1}=\xi\left(\begin{array}{cccc}
0 & v p_{-} & 0 & v_{3} p_{+} \\
v p_{+} & 0 & \xi \gamma_{1} & 0 \\
0 & \xi \gamma_{1} & 0 & v p_{-} \\
v_{3} p_{-} & 0 & v p_{+} & 0
\end{array}\right)
$$

where $p_{ \pm}=p_{x} \pm i p_{y}, v=\sqrt{3} a \gamma_{0} /(2 \hbar)$ and $v_{3}=$ $\sqrt{3} a \gamma_{3} /(2 \hbar)$, while $\xi=+1$ for the valley $\mathbf{K}$ and $\xi=-1$ for the valley $\mathbf{K}^{\prime}(a=0.246 \mathrm{~nm}$ is the lattice constant in the honeycomb lattice). The strength of the trigonal warping is desribed by the parameter $\beta=v_{3} / v=\gamma_{3} / \gamma_{0}$. According to previous studies $20.31 .33 \beta \approx 0.1$.

The four eigenvalues of the Hamiltonian (11) as functions of the wave number $\mathbf{k}=k(\cos \varphi, \sin \varphi)$ are given by

$$
\begin{aligned}
& E_{n}^{2}(k, \varphi)=\frac{\gamma_{1}^{2}}{2}\left[1+\tilde{k}^{2}\left(\beta^{2}+2\right)+(-1)^{n} \Gamma\right], \text { where } \\
& \Gamma=\sqrt{1-2 \tilde{k}^{2}\left(\beta^{2}-2\right)+\tilde{k}^{4} \beta^{2}\left(\beta^{2}+4\right)+8 \tilde{k}^{3} \beta \cos 3 \varphi}
\end{aligned}
$$

where $n=1,2$, while the rescaled wave number is $\tilde{k}=k \gamma_{1} /(\hbar v)$. Owing to the $\cos 3 \varphi$ term the eigenvalues

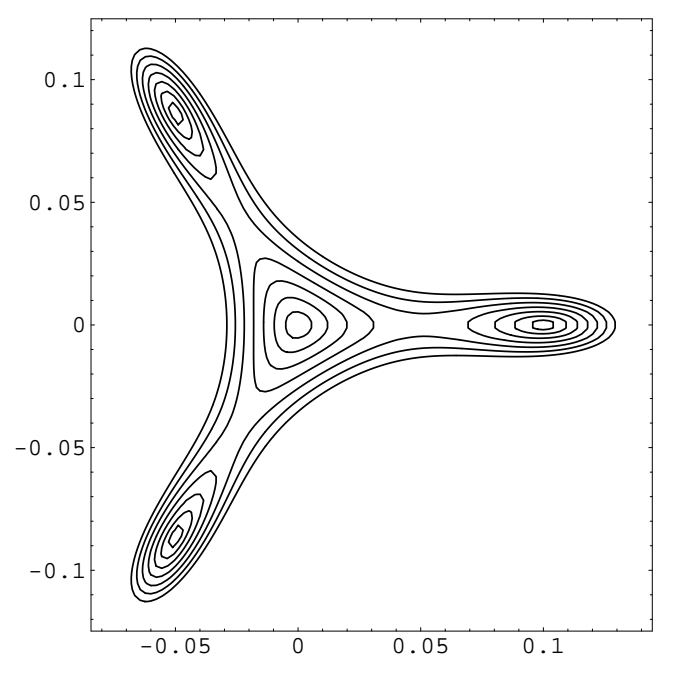

FIG. 1: Constant energy lines (in units of $\gamma_{1}$ ) of the dispersion relation of the positive eigenvalue $E_{1}$ in the $\left(\tilde{k}_{x}, \tilde{k}_{y}\right)$ plane around the $\mathbf{K}$ point of the Brillouin zone (at the origin in this figure). Here $\beta=0.1$ and the contour lines are plotted equidistantly with the most outer contour line corresponding to energy $2 E_{L}$.

are three-fold rotational invariant for finite $\beta$. The eigenvalues $\pm E_{1}$ become zero at the $\mathbf{K}$ point of the Brillouin zone, ie, at $\tilde{k}=0$, and at the center of the three pockets located at $\tilde{k}=\beta$ and $\varphi=0,2 \pi / 3,4 \pi / 3$. Around these zeros the constant energy lines are distorted as shown in Fig. 1. This is called trigonal warping. At moderate energy, direct hopping between $A_{1}$ and $B_{2}$ leads to trigonal warping of the constant energy lines about each valley, but at an energy $E$ less than the Lifshitz energy
$E_{L}=\gamma_{1} \beta^{2} /\left(4+\beta^{2}\right) \approx 1 \mathrm{meV}$, the effect of trigonal warping is dramatic. It leads to a Lifshitz transition 34 : the constant energy line is broken into four pockets, which can be referred to as one central and three leg parts. For $v_{3} \ll v$, ie, $\beta \ll 1$ we find that the separation of the 2D Fermi line into four pockets would take place for very small carrier densities $n<n_{L} \sim 1 \times 10^{11} \mathrm{~cm}^{-2}$. For $n<n_{L}$, the central part of the Fermi surface at energy $E$ is approximately circular with area $\mathcal{A}_{\mathrm{c}} \approx \pi E^{2} /\left(\hbar v_{3}\right)^{2}$, and each leg part is elliptical with area $\mathcal{A}_{\ell} \approx \frac{1}{3} \mathcal{A}_{\mathrm{c}}$. For $E \ll E_{L}$ the dispersion relation are linear in $k$. A similar structure of the constant energy lines can be seen around the $\mathbf{K}^{\prime}$ point. For $\beta=0$ there is no trigonal warping, ie, the eigenvalues are rotational symmetric and the Dirac cones are only at the $\mathbf{K}$ and $\mathbf{K}^{\prime}$ points.

Recently, in self-consistent Born approximation Koshino and Ando $\underline{27}$ have investigated the minimal conductivity for bilayer graphene using an approximated 2 by 2 Hamiltonian which mimics the trigonal warping and is given by

$$
H_{b 2}=g_{2}\left(\begin{array}{cc}
0 & \tilde{p}_{-}^{2}-\tilde{p}_{+} \\
\tilde{p}_{+}^{2}-\tilde{p}_{-} & 0
\end{array}\right)
$$

where the effective coupling constant $g_{2}=\gamma_{1} \gamma_{3}^{2} / \gamma_{0}^{2}$, the rescaled momentums are $\tilde{p}_{ \pm}=\left(p_{x} \pm i p_{y}\right) / p_{0}$ and $p_{0}=$ $2 \hbar \gamma_{1} \gamma_{3} /\left(\sqrt{3} a \gamma_{0}^{2}\right)$.

The simplest effective Hamiltonian valid for $E \ll \gamma_{1}$ and first introduced by McCann and Fal'ko $\underline{20}$ to study the Hall conductivity of bilayer graphene is given by

$$
H_{b 3}=-g_{3}\left(\begin{array}{cc}
0 & p_{+}^{2} \\
p_{-}^{2} & 0
\end{array}\right),
$$

where $g_{3}=v^{2} / \gamma_{1}$ is the effective coupling constant. In this case the trigonal warping is absent. In this work, we calculate the minimal conductivity for all the three Hamiltonians, $H_{b 1}, H_{b 2}$ and $H_{b 3}$.

To find the minimal conductivity for graphene systems we start from the Kubo formula used by Ryu et al. in Ref. 18 which at zero temperature and for dc conductivity (at zero frequency $\omega$ ) is given by

$$
\begin{aligned}
& \sigma_{\mu \nu}^{\min }=n_{s} n_{v} \lim _{\eta \rightarrow 0} \sigma_{\mu \nu}(\eta), \text { where } \\
& \sigma_{\mu \nu}(\eta)=-\delta_{\mu \nu} \frac{\hbar}{4 \pi} \int \frac{d^{2} \mathbf{r}}{S} \int d^{2} \mathbf{r}^{\prime} \Sigma_{\mu \nu}\left(\mathbf{r}, \mathbf{r}^{\prime} ; E=0, \eta\right), \\
& \Sigma_{\mu \nu}\left(\mathbf{r}, \mathbf{r}^{\prime} ; E, \eta\right)=\operatorname{Tr}\left[G^{\mathrm{A}-\mathrm{R}}\left(\mathbf{r}, \mathbf{r}^{\prime} ; E, \eta\right) j_{\mu}\right. \\
& \left.\quad \times G^{\mathrm{A}-\mathrm{R}}\left(\mathbf{r}, \mathbf{r}^{\prime} ; E, \eta\right) j_{\nu}\right]
\end{aligned}
$$

Here $(\mu, \nu)=x, y$, the spin degeneracy is $n_{s}=2$, the valley degeneracy corresponding to the valley $\mathbf{K}$ and $\mathbf{K}^{\prime}$ is $n_{v}=2$, the area of the sample is $S$, while

$$
G^{\mathrm{A}-\mathrm{R}}\left(\mathbf{r}, \mathbf{r}^{\prime} ; E, \eta\right)=G^{-}\left(\mathbf{r}, \mathbf{r}^{\prime} ; E, \eta\right)-G^{+}\left(\mathbf{r}, \mathbf{r}^{\prime} ; E, \eta\right) .(5
$$

The trace is taken over the spinor indices and for systems with translation invariance, the single-particle Green's 
functions are given by

$$
\begin{aligned}
G^{ \pm}\left(\mathbf{r}_{1}, \mathbf{r}_{2} ; E, \eta\right) & =\int \frac{d^{2} \mathbf{k}}{(2 \pi)^{2}} e^{i \mathbf{k}\left(\mathbf{r}_{2}-\mathbf{r}_{1}\right)} G^{ \pm}(\mathbf{k} ; E, \eta),(5 \mathrm{e}) \\
G^{ \pm}(\mathbf{k} ; E, \eta) & =[E \pm i \eta-H(\mathbf{k})]^{-1}, \\
H(\mathbf{k}) & =H(\mathbf{p}=\hbar \mathbf{k}),
\end{aligned}
$$

and the current operator is

$$
j_{\mu}=i \frac{e}{\hbar}\left[H, r_{\mu}\right]=\frac{e}{\hbar} \frac{\partial H(\mathbf{k})}{\partial k_{\mu}} .
$$

The above expression (5b) for the conductivity can be simplified using the identity

$$
(-z-H)^{-1}-(z-H)^{-1}=-2 z\left(z^{2}-H^{2}\right)^{-1} .
$$

Then with $z=i \eta$ we can rewrite $\sigma_{\mu \nu}(\eta)$ in Eq. (5b) as

$$
\begin{aligned}
\sigma_{\mu \nu}(\eta) & =\delta_{\mu \nu} \frac{2 e^{2}}{h} \eta^{2} I(\eta), \text { where } \\
I(\eta) & =\int \frac{d^{2} \mathbf{k}}{(2 \pi)^{2}} \operatorname{Tr}\left[\left[\eta^{2}+H^{2}(\mathbf{k})\right]^{-1} \frac{\partial H(\mathbf{k})}{\partial k_{\mu}}\right. \\
& \left.\times\left[\eta^{2}+H^{2}(\mathbf{k})\right]^{-1} \frac{\partial H(\mathbf{k})}{\partial k_{\nu}}\right] .
\end{aligned}
$$

This expression of the conductivity is valid for translational invariant systems.

Before we turn to the case of the bilayer it is instructive to see how the expression (7b) works for single layer graphene. In this case the Hamiltonian is given by

$$
H_{s}(\mathbf{k})=g_{s}\left(\begin{array}{cc}
0 & k_{-} \\
k_{+} & 0
\end{array}\right) \text {, }
$$

where $g_{s}=\hbar v$ and $k_{ \pm}=k_{x} \pm i k_{y}$. The integrand in Eq. (7b) can easily be calculated using the polar coordinates $\mathbf{k}=k(\cos \varphi, \sin \varphi)$ and one finds

$$
I(\eta) \equiv I_{s}(\eta)=\int_{0}^{\infty} \frac{2 g_{s}^{2} k}{\left(g_{s}^{2} k^{2}+\eta^{2}\right)^{2}} \frac{d k}{2 \pi}=\frac{1}{2 \pi \eta^{2}},
$$

which is independent of the coupling constant $g_{s}$. Note that the main contribution in the integral $I(\eta)$ comes from the vicinity of $k=0$, therefore the integral over $k$ can be extended to infinity $\underline{15}$. Then from Eqs. (7a) and (5a) we obtain the well-known universal value of the minimal conductivity for single layer graphene: $\sigma_{x x}^{\min }=$ $(4 / \pi)\left(e^{2} / h\right)^{11,13,14,15,16,17,18}$.

We now consider the bilayer graphene taking into account the effect of the trigonal warping. For bilayer with Hamiltonian (11) the current operators defined by Eq. (5h) have a simple form

$$
\begin{aligned}
& j_{x}=\xi \frac{e v}{\hbar}\left(\begin{array}{cccc}
0 & 1 & 0 & \beta \\
1 & 0 & 0 & 0 \\
0 & 0 & 0 & 1 \\
\beta & 0 & 1 & 0
\end{array}\right) \\
& j_{y}=i \xi \frac{e v}{\hbar}\left(\begin{array}{cccc}
0 & -1 & 0 & \beta \\
1 & 0 & 0 & 0 \\
0 & 0 & 0 & -1 \\
-\beta & 0 & 1 & 0
\end{array}\right) .
\end{aligned}
$$

The integral $I(\eta) \equiv I_{b 1}(\beta, \eta)$ in Eq. (7b) will depend on $\beta$. It can be shown that $\sigma_{x x}=\sigma_{y y}$, therefore it is convenient to calculate $\sigma_{x x}=\left(\sigma_{x x}+\sigma_{y y}\right) / 2$. Using the polar coordinates $\mathbf{k}=k(\cos \varphi, \sin \varphi)$ and rescaling the variables $k$ and $\eta$ as $k \rightarrow k \hbar v / \gamma_{1}$ and $\eta \rightarrow \eta / \gamma_{1}$, a straightforward algebra yields for the case of $\xi=+1$ (valley $\mathbf{K}$ ):

$$
\begin{aligned}
& I_{b 1}(\beta, \eta)=\int_{0}^{\infty} \int_{0}^{2 \pi} k \frac{A+B \cos 3 \varphi}{(C+D \cos 3 \varphi)^{2}} \frac{d \varphi}{2 \pi} \frac{d k}{2 \pi} \\
& \quad A=k^{4}\left(2+5 \beta^{2}\right) \\
& \quad+\left(1+\eta^{2}\right)\left[4 k^{2}\left(1+\beta^{2}\right)+2 \eta^{2}+\beta^{2}\left(1+\eta^{2}\right)\right]( \\
& B=4 k^{3} \beta \\
& \quad C=k^{4}+\eta^{4}+\eta^{2}+k^{2}\left[2 \eta^{2}+\beta^{2}\left(1+\eta^{2}\right)\right] \\
& D=-2 k^{3} \beta
\end{aligned}
$$

This expression has a three-fold rotational symmetry as should be for trigonally warped bilayer graphene. It can be shown that for $\xi=-1$ (valley $\mathbf{K}^{\prime}$ ) we have the same results. The conductivity is two-fold degenerate according to the valleys, ie, $n_{v}=2$.

The integral over $\varphi$ can be performed analytically and $I_{b 1}(\beta, \eta)$ becomes

$$
I_{b 1}(\beta, \eta)=2 \int_{0}^{\infty} k \frac{A C-B D}{\left(C^{2}-D^{2}\right)^{3 / 2}} \frac{d k}{2 \pi} .
$$

Without trigonal warping, ie, for $\beta=0$ one finds

$$
\begin{gathered}
I_{b 1}(\beta=0, \eta)= \\
\int_{0}^{\infty} \frac{d k}{2 \pi} \frac{4 k\left(k^{4}+\eta^{4}+\eta^{2}+2 k^{2}+2 k^{2} \eta^{2}\right)}{\left(k^{4}+\eta^{4}+\eta^{2}+2 k^{2} \eta^{2}\right)^{2}}=\frac{1}{\pi \eta^{2}} .
\end{gathered}
$$

Thus, using Eqs. (7a) and (5a) the minimal conductivity for bilayer graphene without trigonal warping is $\sigma_{x x}^{\min }(\beta=0)=(8 / \pi)\left(e^{2} / h\right)$. This result has been derived first in Ref. 17 in a different way, and subsequently by Snyman and Beenakker in Ref. 29 using the Landauer approach.

After a tedious calculation the integral in Eq. (12) for finite value of $\beta$ can be performed yielding

$$
\begin{aligned}
I_{b 1}(\beta, \eta)= & \frac{1}{4 \pi \eta^{2}}\left(12-\frac{127+145 \beta^{2}+38 \beta^{4}}{\beta^{6}+\beta^{4}} \eta^{2}\right) \\
& +O(\ln \eta)+O\left(\eta^{2}\right) .
\end{aligned}
$$

Again, using Eqs. (7a) and (5a) we find a remarkable result, namely the minimal conductivity for bilayer graphene with trigonal warping takes a universal value $\sigma_{x x}^{\min }(\beta)=(24 / \pi)\left(e^{2} / h\right)$ independent of the stregth $\beta$ of the warping. This is our central result in this paper. This value is six times as large as the conductivity in single layer graphene. It is suprising that $\sigma_{x x}^{\min }(\beta)$ is not a continous function around $\beta=0$. Indeed, as we have seen $\sigma_{x x}^{\min }(\beta=0)=(8 / \pi)\left(e^{2} / h\right)$, while for any finite values of $\beta$ it is three times larger. This non-analitic behaviour of $\sigma_{x x}^{\min }(\beta)$ at $\beta=0$ is a consequence of the fact that the 
minimal conductivity results from the electronic dynamics in the limit of zero density $n \rightarrow 0$. For any non-zero $\beta$, such density is always below the Lifshitz density $n<n_{L}$ where the 2D Fermi line around each valley forms four separate pockets, whereas for $\beta=0$, the Lifshitz transition does not occur and there is always a single Fermi line at each valley.

In the frame work of self-consistent Born approximation the same result was predicted by Koshino and Ando 27 using the Hamiltonian $H_{b 2}$ given by Eq. (3). Note that in this Hamiltonian there is no adjustable parameter for the strength of the trigonal warping like $\beta$ for Hamiltonian (11). The effective coupling constant $g_{2}$ drops out in Eq. (7b), therefore in this model the trigonal warping is built in without the possibility to change its strength. Using Eq. (77) we repeat the calculation with the Hamiltonian (3) and find

$$
I(\eta) \equiv I_{b 2}(\eta)=\frac{1}{4 \pi \eta^{2}}\left(12-127 \eta^{2}\right)+O(\ln \eta)+O\left(\eta^{2}\right) .
$$

Thus, the minimal conductivity takes the same universal value $\sigma_{x x}^{\min }(\beta)=(24 / \pi)\left(e^{2} / h\right)$ as that for the Hamiltonian $H_{b 1}$ given by (1).

Finally, we calculate the minimal conductivity using the simplest Hamiltonian $H_{b 3}$ given by Eq. (4). Then the integral in (7b) becomes

$$
I(\eta) \equiv I_{b 3}(\eta)=\int_{0}^{\infty} \frac{d k}{2 \pi} \frac{8 g_{3}^{2} k^{3}}{\eta^{2}+g_{3}^{2} k^{4}}=\frac{1}{\pi \eta^{2}}
$$

Thus, the minimal conductivity takes the same universal value $\sigma_{x x}^{\min }=(8 / \pi)\left(e^{2} / h\right)$ as that for Hamiltonian (1) with $\beta=0$.

In summary, we compared the minimal conductivity in bilayer graphene obtained from three different effective Hamiltonians used in the literature. We found that for the case when the trigonal warping is absent, the conductivity is always two times larger, while in the presence of trigonal warping it is six times larger than that for single layer graphene and is independent of the strength of the warping. Our universal results suggests that the conductivity has a topological origin, which can be a further research topic in the future.

We gratefully acknowledge discussions with C. W. J. Beenakker, E. McCann, and V. Fal'ko. This work is supported partially by European Commission Contract No. MRTN-CT-2003-504574 and the Hungarian Science Foundation OTKA 046129.
${ }^{1}$ K. S. Novoselov, A. K. Geim, S. V. Morozov, D. Jiang, Y. Zhang, S. V. Dubonos, I. V. Grigorieva, and A. A. Firsov, Science 306, 666 (2004); K. S. Novoselov, A. K. Geim, S. V. Morozov, D. Jiang, M. I. Katsnelson, I. V. Grigorieva, S. V. Dubonos, and A. A. Firsov, Nature 438, 197 (2005).

2 Y. Zhang, J. P. Small, M. E. S. Amori, and P. Kim, Phys. Rev. Lett. 94, 176803 (2005); Y. Zhang, Y.-W. Tan, H. L. Stormer, and P. Kim, Nature 438, 201 (2005).

3 K. S. Novoselov, E. McCann, S. V. Morozov, V. I. Fal'ko, M. I. Katsnelson, U. Zeitler, D. Jiang, F. Schedin, A. K. Geim, Nature Physics 2, 177 (2006).

4 M. I. Katsnelson, Materials Today 10, 20 (2007).

5 M. I. Katsnelson and K. S. Novoselov, cond-mat/0703374

6 A. K. Geim and K. S. Novoselov, Nature Materials 6, 183 (2007).

7 E. Fradkin, Phys. Rev. Rev. B 63, 3263 (1986); P. A. Lee, Phys. Rev. Lett. 71, 1887 (1993); E. V. Gorbar, V. P. Gusynin, V. A. Miransky, and I. A. Shovkovy, Phys. Rev. B 66, 045108 (2002).

8 K. Ziegler, Phys. Rev. Lett. 97, 266802 (2006).

${ }^{9}$ L. A. Falkovsky and A. A. Varlamov, cond-mat/0606800.

10 K. Nomura and A. H. MacDonald, cond-mat/0606589.

11 V. P. Gusynin and S. G. Sharapov, Phys. Rev. Lett. 95, 146801 (2005).

12 V. P. Gusynin and S. G. Sharapov, Phys. Rev. B 73, 245411 (2006).

13 N. M. R. Peres, F. Guinea, and A. H. Castro Neto, Phys. Rev. B 73, 125411 (2006).

14 M. I. Katsnelson, Eur. Phys. J. B 51, 157 (2006).

15 P. M. Ostrovsky, I. V. Gornyi, and A. D. Mirlin, Phys. Rev. B 74, 235443 (2006).

16 J. Tworzydło, B. Trauzettel, M. Titov, A. Rycerz, C.W.J.
Beenakker, Phys. Rev. Lett. 96, 246802 (2006).

17 J. Cserti, Phys. Rev. B 75, 033405 (2007).

18 S. Ryu, C. Mudry, A. Furusaki, and A. W. W. Ludwig, cond-mat/0610598.

19 F. Miao, S. Wijeratne, U. Coskun, Y. Zhang, and C. N. Lau, cond-mat/0703052

20 E. McCann and V. I. Fal'ko, Phys. Rev. Lett. 96, 086805 (2006).

21 E. McCann, Phys. Rev. B 74, 161403 (2006).

22 E, V. Castro, et al. cond-mat/0611342.

23 T. Ohta, A. Bostwick, T. Seyller, K. Horn, and E. Rotenberg, Science 313, 951 (2006).

24 D. S. L. Abergel and V. I. Fal'ko, cond-mat/0610673

25 J. Nilsson and A. H. Castro Neto, cond-mat/0612470

26 T. Ludwig, cond-mat/0701045.

27 M. Koshino and T. Ando, Phys. Rev. B 73, 245403 (2006).

28 M. I. Katsnelson, Eur. Phys. J. B 52, 151 (2006).

29 I. Snyman and C. W. J. Beenakker, Phys. Rev. B 75, 045322 (2007).

${ }^{30}$ K. Kechedzhi, V. I. Fal'ko, E. McCann, and B. L. Altshuler, cond-mat/0701690.

31 W. W. Toy, M. S. Dresselhaus and G. Dresselhaus, Phys. Rev. 109, 272 (1958).

32 A. Misu, E. Mendez, and M. S. Dresselhaus, J. Phys. Soc. Jpn. 47, 199 (1979).

33 R. E. Doezema, W. R. Datars, H. Schaber, and A. Van Schyndel, Phys. Rev. B 19, 4224 (1979).

34 A. A. Abrikosov, Fundamentals of the Theory of Metals, Elsevier Science Publishers B. V., North-Holland, Amsterdam, 1988. 